\title{
Free-electron transparent metasurfaces with controllable losses for broadband light manipulation with nanometer resolution
}

Marcella Bonifazi ${ }^{1 \dagger}$, Valerio Mazzone ${ }^{1 \dagger}$, Ning $\mathrm{Li}^{1}$, Yi $\operatorname{Tian}^{1}$, Andrea Fratalocchi ${ }^{1 *}$

${ }^{1}$ PRIMALIGHT, Faculty of Electrical Engineering; Applied Mathematics and Computational Sci-

ence, King Abdullah University of Science and Technology, Thuwal 23955-6900, Saudi Arabia

${ }^{\dagger}$ These authors contributed equally to this work.

Controlling broadband light in nanoscale volumes of matter is a desired goal in nanophotonics. Metastructures tackle this problem by engineering subwavelength patterns in nanostructured materials. The state of the art of this technology reaches footprints within $50 \mathbf{n m}$ with plasmonic nanostructures. Scaling down these values is challenging, especially in low loss and inexpensive dielectric materials. We here introduce a new class of metasurfaces, "printed" point to point by free-electron waves of suitable intensity and created by altering the resonant atomic transition of inexpensive photosensitive materials. We demonstrate that with this approach it is possible to directly write a desired distribution of refractive index $n(\mathbf{r})$ and extinction coefficient $k(\mathbf{r})$ with a resolution equal to the focusing accuracy of the electron beam, theoretically limited to the single nanometer. We illustrate an application of this technology in structural coloration. Currently, the best results with the highest resolution are obtained with plasmonic heterostructures at 127000 DPI, with $50-200 \mathbf{n m}$ structures and chromaticity ranging from blue to yellow. We experimentally show that free-electron meta- 
surfaces could generate the complete spectrum of colors of the Cyan, Yellow, Magenta, and Black (CYMK) system with resolutions up to 256000 DPI, and nanostructures of $10 \mathrm{~nm}$ radius by using a single inexpensive layer of transparent material. This platform could enable a new generation of low cost transparent media supporting ultra-dense optical circuitry for broadband light control.

\section{Introduction}

Metasurfaces are engineered structures for controlling light in different applications including wavefront shaping ${ }^{11,19,24,26,28}$, structural coloration ${ }^{7,8,12,13,15,17,18,25,27,31-34,36-38}$, hyperbolic structures ${ }^{20}$, bio-imaging ${ }^{35}$ and lensing ${ }^{4,14,21,29,30}$. Metastructures are typically realized by engineering subwavelength patterns of different shapes in metal and/or dielectric heterostructures. The smaller the size of the pattern, the better the resolution and the controllability of the material response over a larger frequency bandwidth. This scaling trend is visually illustrated in Figure 1a, which summarizes the main results published in the last few years along different lines of research.

Plasmonics currently supports structures with the smallest footprint, but in highly absorbing structures that are not transparent ${ }^{1}$. Metasurfaces constituted by dielectric, low-loss materials are currently above characteristic dimensions of $200 \mathrm{~nm}$. Creating transparent nanoscale materials providing similar — or better- performances is now at the center of huge research efforts ${ }^{16}$. The large density of structures observed in Fig. 1a in lower-left corner witnesses the challenges of present technological platforms to reduce circuit dimensions below characteristic lengths around $100 \mathrm{~nm}$. If these limits could be overcome, we could create platforms to control broadband light 
from inexpensive transparent structures with dense optical circuitry possessing nanoscale —and smaller- footprints.

In this article we explore a strategy that takes advantage from atomic interactions between a transparent photosensitive material and intense free-electrons beams. This approach allows us to reach resolution of $20 \mathrm{~nm}$ (Fig. 1a), without requiring the use of metallic structures and by just using inexpensive transparent dielectrics.

Traditionally, electron-beam technology is employed in the creation of binary patterns that are used as a mask for the etching of substrates or for the deposition of metal/dielectric layers ${ }^{3}$. With this approach it is difficult to control the material response below $50 \mathrm{~nm}$, because of the necessary resolution to create the required geometrical patterns. Our study shows that by carefully controlling the flux of free-electrons of $E=50 \mathrm{keV}$ energy, it is possible to access a sub-nanoscale regime of electrons-matter interaction, in which the resonant atomic transition of a photosensitive material are controllably changed, printing point-to-point a desired distribution of refractive index and absorption coefficient in the entire visible range and from an inexpensive medium. Experiments confirmed by theory demonstrate that both the real and imaginary part of the refractive index are easily controlled in circuits with footprints equal to $20 \mathrm{~nm}$.

We illustrated an application of this technology in structural coloration, a topic of large contemporary interest for the many possible applications in smart fabrics, high resolution ink-free color printing, anti-counterfeiting technologies and adaptive camouflage materials ${ }^{7,8,12,13,15,17,18,25,27,31-34,36-38}$. The state of the art with the highest resolution is represented by heterostructures composed by polymers covered by aluminum or germanium ${ }^{38}$ and by plasmonic metasurfaces with gold-aluminum 
nanostructures of $50-140 \mathrm{~nm}$ size ${ }^{18,37}$. These structures achieve a maximum resolution of 127000 DPI, with a limited chromaticity spectrum ranging from blue to yellow. Images with complete chromaticity have been realized with $\mathrm{Si} / \mathrm{Si}_{3} \mathrm{~N}_{4} / \mathrm{Si}$ heterostructures, reporting a resolution of 40000 DPI on an area of $300 \mu \mathrm{m} \times 200 \mu \mathrm{m}$ size ${ }^{6}$. Our experiments demonstrate that free-electron metastructures can generate the full chromaticity spectrum including cyan, magenta, yellow, black (CMYK) colors with resolution up to 254000 DPI, printing large scale images on $280 \mu \mathrm{m}$ x $196 \mu \mathrm{m}$ from a single transparent layer without the use of dyes, pigments or metals.

\section{Results}

Figure $1 b-d$ illustrates the general process for the realization of a free-electron metasurface. A thin film of photosensitive material is initially deposited on top of a flat silicon reflector and then irradiated with free-electrons with different doses and three dimensional patterns. The selected thickness of transparent material was optimized to find the best compromise between the gamut of chromaticity generated and the achievable DPI resolution. We experimentally found that the optimal value for this parameter is $350 \mathrm{~nm}$, which guarantees the realization of widest range of colors, thanks to the variation in the EB dose, with the highest resolution. We assessed that lower thicknesses yield to narrower gamut of chromaticity, since the EB does not irradiate enough amount of material. Higher thicknesses, instead, reduced the DPI resolution and the attainable spectrum of colors, since they make it impossible to nanofabricate disks of $40 \mathrm{~nm}$ radius.

In the area where the beam is strongly focused, the material changes its molecular structure and becomes soluble by alcohol solutions (see Methods), creating a 3D pattern of air holes as in con- 
ventional e-beam lithography (Fig. 1c). In the remaining volume, scattered electrons at lower intensity provide a weaker atomic interaction that shifts the resonant transitions of the electronic structure of the material (Fig. 1d), resulting in a platform with two degrees of freedom, irradiation patterns and free-electron doses, to controllably write on the material a distribution of refractive index $n(\mathbf{r}, \omega)$ and extinction coefficient $k(\mathbf{r}, \omega)$.

The transparent material used in this study is Poly-Methyl-Methacrylate (PMMA), which is a widely employed photoresists ${ }^{10}$. The free-electrons beam is generated in a Crestec CABL-9520C, which is an advanced e-beam (EB) nanofabrication facility (see Methods).

In the first experiment we characterize the response of free-electron metasurfaces created with three dimensional patterns consisting of random distributions of nanodisks, each of radius $r$ and thickness $d$ ranging from $50 \mathrm{~nm}$ to $300 \mathrm{~nm}$, arranged in disordered configurations over an area of $20 \mu \mathrm{m} \times 20 \mu \mathrm{m}$. The use of disordered configurations of holes is preferable over periodic structures as the former maximizes the entropy of different configurations for the irradiation pattern. In a periodic structure containing $N$ elements, the degrees of freedom available to change configuration are fixed to 6 (three displacements unit vectors for the lattice cell, and three periodic lengths along $\mathrm{x}, \mathrm{y}$, and $\mathrm{z}$ ) while in a disordered system this number grow as $3 \cdot N$ (three coordinates specifying the independent position of each element of the system).

Different random patterns are generated by randomizing the position of a periodic distribution of nanodisks with radius $r=40 \mathrm{~nm}$ and chosen DPI. The latter is calculated from the average distance between the center of two neighbouring nanodisks (the pitch) $a 0=r / f_{v}$, defined as the ratio between the radius $r$ of the disks and the disk filling fraction $f_{v}$. The resulting $D P I=(2.54 \mathrm{~cm}) / a 0$ 
is then obtained from the number of circles that are printed in one inch.

In the randomization procedure, we imposed the constraint that each nanodisk does not overlap with another. With this condition the filling fraction and the DPI of the disordered configuration are preserved and are the same as in the original structure.

To illustrate how the patterns change at different filling fractions $f_{v}$ and radii, we collected in Supplementary Fig. 1 scanning electron microscope (SEM) images of the samples at $r=150 \mathrm{~nm}$, $r=200 \mathrm{~nm}$ and $f_{v}$ ranging from 0.125 to 0.375 . In supplementary Fig. 2 images of the original computer generated pattern (a) and of a fabricated metasurface (b) are shown for comparison. Due to the dielectric nature of the materials used in the fabrication, a lighter area around each patterned hole is present. This effect is due to the accumulation of electrons on the edge of the nanostructures during the acquisition of the SEM images.

The ability to precisely transfer the pattern to the transparent material results from the properties of the free-electron beam used, and the parameters chosen to expose the sample. The EB facility employed in this work generates free electrons beams focused up to a characteristic size of $2 \mathrm{~nm}$ and with an equivalent De Broglie wavelength of $5.49 \mathrm{pm}$. This enables the possibility to control the overall pattern with atomic scale precision, while modifying the PMMA characteristics with nanometer accuracy.

In our study, we did not apply any proximity correction on the EB impinging on the sample. With proximity corrections, the irradiation pattern is automatically modified by the EB station to avoid cross talking effects, i.e., irradiation effects from one area to another. These effects are normally unwanted, as they are believed to be uncontrollable. In this work, conversely, we demonstrate 
that these effects can be beneficial, if properly enabled, and can lead to new nanoscale regimes of light-matter interactions.

The dose $D$ of the electron beam is expressed through:

$$
D=\frac{t_{e} \cdot I}{A}
$$

with $t_{e}$ the exposure time, $I$ the free-electron current and $A$ the exposed area. In our experiments, we regulated the electron beam dose by changing the exposure time $t_{e} \in[0.1 \mu s, 2 \mu s]$ at a fixed current density $I / A$ (see Methods).

After the exposure, we characterized the generated metasurface by reflectivity measurements at normal incidence (see Methods). Resulting spectra are illustrated in Fig. 2a-b. In these figures, the color of each spectra shows the chromaticity visually observed in the generated metasurface. Figure 2a summarizes on the left inset the complete palette of colors generated in the free-electron metasurfaces. Figure 2c shows the corresponding chromaticity in a CIE 1976 diagram.

The generated metasurfaces are able to create the full CMYK system of colors, with colors spanning from green to black with the exposure increasing from $t_{e}=0.1 \mu$ s to $t_{e}=2.0 \mu \mathrm{s}$ (Fig. 2a) and from blue to yellow when the filling fraction increases from $f_{v}=0.125$ to $f_{v}=0.375$ (Fig. b). Representative chromaticity ranging from green to blue via pink, red, violet and black completes a closed circle around the white point (Fig. 2c). These metasurfaces are also able to create the red color, which is traditionally challenging to obtain with structures and usually obtained with dyes and pigments ${ }^{23}$. The surface is also capable to obtain the black color, despite the original transparency of the PMMA layer.

To ascertain the resolution achievable by these metasurfaces, we performed experiments by fabri- 
cating $20 \mu \mathrm{m} \times 20 \mu \mathrm{m}$ squares samples with constant exposure time, different radius $r$ and filling fraction $f_{v}$, starting from nanodisks with $r=200 \mathrm{~nm}$ and $f_{v}=0.8$ and decreasing to nanodisks with $r=10 \mathrm{~nm}$ and $f_{v}=0.1$ (Fig. 2d-n). The corresponding resolution is expressed in terms of number of dots per inch (DPI).

The experiments demonstrate that this technique can generate different colors with resolutions as high as $254000 \mathrm{DPI}$, corresponding to a pitch of $100 \mathrm{~nm}$ obtained with a radius of $r=10 \mathrm{~nm}$ and a filling fraction of $f_{v}=0.1$. These results show that cross-talking effects are controlled by means of the geometrical parameters of the generated pattern $\left(r\right.$ and $\left.f_{v}\right)$ and the exposure dose used. In free electrons metasurfaces fabricated with irradiation patterns of $10 \mathrm{~nm}$ radius nanodisks (Fig. 2n) the cross talking effect has a characteristic length lower than $100 \mathrm{~nm}$. This observation stems from the fact that there is no cross exposure between holes and their shape is preserved. From different combination of these parameters we manage to control the overall pattern conserving the shapes and fine tuning the exposure of the PMMA to obtain the desired color.

The printing resolution can be further increased by reducing the period of the initial (nonrandom) array and the disk radius $r$. This possibility is not investigated here as the value of $r=10 \mathrm{~nm}$ is the smallest nanodisk that can be realized with the EB station used in this work.

To theoretically demonstrate the generation of a free-electron induced metastructure, we begin by showing that the results of Fig. 2 cannot be generated by the Si/PMMA structure in which the PMMA maintains its original optical response. In Supplementary Fig. 3 we perform a campaign of dispersive Finite Difference Time Domain (FDTD) simulations 9,22 comparing the spectra predicted by FDTD against the experiments of Fig. 2, which comprise the largest variation of colors. 
In the FDTD simulations we considered the effect of different free-electrons doses by assigning different depths to the holes constituting the irradiation pattern. This mimics the process in which the PMMA material is progressively removed when exposed to higher energy fluxes of free-electrons from the EB radiation. Theoretical spectra are calculated by employing the experimental refractive index of Silicon and PMMA and the patterns of air inclusions experimentally generated in these structures and acquired by SEM images. The increasing dose of electrons is simulated by increasing the depth $d$ of the patterns (Supp. Fig. 3a-d, insets) from $d=0$ (no PMMA is removed) to $d=350 \mathrm{~nm}$ (all PMMA is removed inside the nanodisks). Supplementary Fig. 3a shows that this model correctly reproduces the material response when the PMMA is irradiated with low doses (short exposure time). Once higher doses are employed to interact with the photosensitive layer, FDTD spectra and actual measurements become completely different (Supp. Fig. 3b-d).

A further demonstration that the response of Fig. 2 cannot be generated from the original materials is obtained via Maxwell-Garnett model ${ }^{5}$, in which the effective dielectric constant of the medium is expressed as: $\epsilon_{e f f}=\epsilon_{m} \frac{2 \delta_{i}\left(\epsilon_{i}-\epsilon_{m}\right)+\epsilon_{i}+2 \epsilon_{m}}{2 \epsilon_{m}+\epsilon_{i}-\delta_{i}\left(\epsilon_{i}-\epsilon_{m}\right)}$, where $\epsilon_{m}$ is the the electric permittivity of the PMMA, $\epsilon_{i}=1$ and $\delta_{i}$ is the volume fraction of the air inclusions. The Maxwell-Garnett model explores all the possible geometrical configurations that are described by a fixed volume ratio air/PMMA, while keeping the material dielectric constants and the thickness of the medium fixed (in this case at $d=350 \mathrm{~nm}$ ). Supplementary Fig. 3e compares these predictions (light blue squares) with FDTD results (red triangles) and experiments (blue circles and yellow dashed dotted line). This analysis shows that FDTD and Maxwell-Garnett model are consistent (FDTD spectra moves along the curve predicted by Maxwell-Garnett) and shows that both models cannot explain the experi- 
mental outcomes from the nanostructuring of the PMMA layer. Different effective thicknesses $d$ of PMMA have also been considered in the Maxwell-Garnett approximation, taking into account potential fluctuation in the thickness of PMMA deposited by spin-coating (Supp. Fig. 4a-d). In our analysis we varied the thickness $d$ from $d=100 \mathrm{~nm}$ (Supp. Fig. 4a) to $d=300 \mathrm{~nm}$ (Supp. Fig. 4d), and considered all the possible filling fraction of air inclusions. In all these cases the predictions are unable to reproduce the experiments.

This analysis shows that the experimental results are generated from a complex modification of the PMMA layer, in which the material response is modified by the impinging free-electrons as a function of the applied dose. To study this process, we used an effective medium approach based on a simple quantum model arising from linear response theory ${ }^{2}$. For an isotropic medium, the real and imaginary part of the refractive index $n(\mathbf{r}, \omega)+i k(\mathbf{r}, \omega)=\sqrt{1+\chi(\mathbf{r}, \omega)}$ are defined through the linear susceptibility $\chi(\mathbf{r}, \omega)=\chi_{r}(\mathbf{r}, \omega)+i \chi_{i}(\mathbf{r}, \omega)$ resulting from electronic transitions between a ground state $|0\rangle$ and $|1\rangle,|2\rangle, \ldots,|N\rangle$ excited states:

$$
\chi(\mathbf{r}, \omega)=\frac{2 \rho_{0}}{3 \epsilon_{0} \hbar} \sum_{n=1}^{N}\left|\boldsymbol{\mu}_{n 0}\right|^{2}\left(\frac{1}{\omega_{n 0}-\omega-i \gamma_{n 0}}+\frac{1}{\omega_{n 0}+\omega+i \gamma_{n 0}}\right)
$$

with $\rho_{0}$ the atomic number density, $\hbar \omega_{n 0}=E_{n}-E_{0}$ the energy level difference, $\gamma_{n 0}$ damping rates and $\boldsymbol{\mu}_{n 0}=\langle n|\boldsymbol{\mu}| 0\rangle$ the strength of the electric dipole moment $\boldsymbol{\mu}=-e \mathbf{r}$ for the $0 \rightarrow n$ transition. To characterize the spectra of Fig. 2, we assume that each irradiated area responds as a material with refractive index $n$ and extinction coefficient $k$ following from Eq. (2), in which resonant transitions $\omega_{n 0}$, damping factors $\gamma_{n 0}$ and oscillator strengths $f_{n 0}=\frac{2 m \omega_{n 0}\left|\mu_{n 0}\right|^{2}}{3 \hbar e^{2}}$ are modified by the electron beam.

As the PMMA is a transparent dielectric structure, we assume the existence of a single transition 
$(\mathrm{N}=1)$ in the deep UV region (Fig. 3a-b) that influences the material response in the visible and near infrared ${ }^{2}$. This model well represents the experimental response of the PMMA in the entire visible range (Fig. 3c, circle markers). The values of the coefficients $\omega_{10}, \gamma_{10}$ and $f_{10}$ when the material is irradiated by electrons are found by fitting the reflectivity spectra at normal incidence, using the experimental dispersion curve of Si available in the literature (see Methods).

Figure $3 c$ illustrates in details the effects of these changes on the response of the metasurface. The atomic resonance of the PMMA, originally in the deep UV, tends to red-shifts for increasing exposure time, increasing its damping $\gamma_{10}$ factor. This dynamics makes the PMMA structure ultratransparent, with refractive index below 1.2, while increasing its absorption capabilities, thus being able to generate the entire spectrum of visible colors, including the black.

The extinction coefficient $k$ varies from 0 to 0.025 (Fig. 3c, red lines) and, together with the changes in the refractive index $n$, it generates a considerable variation of the chromaticity in the realized samples, as it is shown in the insets of Figures 3e-h, in which the color corresponding to each experimental reflectivity spectra is presented. The result of this analysis matches very well the acquired spectra (Fig. 3d-h), showing that the measured response originates from a PMMA layer in which both the resonant frequency $\omega_{10}$ and the damping factor $\gamma_{10}$ are independently changed with complex modulations patterns at increasing exposure times (Fig. 3h).

The EB irradiation effects on the the PMMA layer are irreversible, as experimentally observed by the stability of the generated colors over a six month observation time. In this period, the visual appearance of the metasurface did not display any appreciable change and the colors were stable. Figure 4a-p illustrates different printing applications with free-electron metasurfaces. In these 
images, we varied exposure time, filling fraction and radius of the nanodisks in different portions of the designs in order to obtain different colors, spanning from magenta to black in the background of the greek letter $\alpha$ design (Fig. 4a-d), and from cyan to orange inside the letter $\beta$ (Fig. 4g-l). Scanning electron microscope images of Fig. 4d,g show the different nanoscale patterns that, combined with different e-beam doses, generate different colors. The patterns follow sharply the profile of the printed shapes from the array of nanodisks with very high resolution.

This is further illustrated in Supplementary Fig. 4a-d, which show the word "light" using the four basic colors Cyan (Supp. Fig. 4a), Magenta (Supp. Fig. 4b), Yellow (Supp. Fig. 4c) and Black (Supp. Fig. 4d) and different languages (Latin, Arabic, Japanese and Chinese) printed at different resolutions: 127000 DPI (Japanese), 55580 DPI (Latin), 36290 DPI (Chinese), and 84670 DPI (Arabic). Figure 41-q shows a large scale nanoprinting cropped representation of the painting by Matisse "La Danse" (reported here in Figure 4k, Henri Matisse, La Dance, 1910, CCSuccession H. Matisse $/ 2019$, ProLitteris, Zrich), on a size of $280 \mu \mathrm{m} \times 196 \mu \mathrm{m}$. SEM analysis of different portions of the image are presented in (Fig.4o-q). We used different number of DPI for the different artistic elements present in the painting, according to the overall distribution of colors and the chromaticity we wanted to reproduce, with an average of 100000 DPI and a maximum of 119250 DPI in the brown areas. The image is printed from a single inexpensive layer of PMMA.

\section{Conclusion}

We have designed, fabricated and characterized a new family of nanomaterials in which the desired distribution of refractive index and losses is printed by free-electrons beams of varying in- 
tensity and radiation patterns. The materials are generated by sub-nanometer interactions between free-electrons and photosensitive materials, which shifts their atomic transitions thus generating an optical response controllable point to point within the focusing accuracy of the free electron beam. We discussed this approach with reference to structural coloration, by using an inexpensive transparent layer of PMMA, demonstrating broadband control of light via the generation of all the colors present in the visible spectrum, including the Cyan, Magenta, Yellow and Black, of the CMYK system. We demonstrate that this technique can reach very high resolutions up to 254000 DPI, overcoming the limits of current techniques, as well as large scale nanoprinting areas with high quality and vivid images, sharp contours and homogeneous colors.

This technique does not involve the use of high-cost metals but it relies on an inexpensive platform composed only by a dielectric back-reflector and a transparent layer of PMMA, which is a very common photosensitive material. In addition, the described coloration technique could be employed in harsh environment, adding on top of the nanostructured material an epoxy protecting layer that will not alter the coloration. This approach can be generalized to the creation of new free-electron metasurfaces for applications that require broadband control of light with dense optical circuitry, including also flexible substrates for wearable optoelectronic components.

\section{Acknowledgements}

This research acknowledges funding from KAUST (Award OSR-2016-CRG5-2995).

Image is used from www.hermitagemusum.org, courtesy of The State Hermitage Museum, St. Petersburg, Russia. 


\section{Methods}

E-metasurface nanofabrication A layer of $350 \mathrm{~nm}$ thick of PMMA 950 A4 (MicroChem) was spin-coated onto $1.5 \mathrm{~cm}$ by $1.5 \mathrm{~cm}$ Silicon substrates. To allow the solvent to evaporate, samples were subsequently baked at $180{ }^{\circ} \mathrm{C}$ for 180 seconds. The fabrication process was performed using Crestec CABL-9520C (http://www.crestec8.co.jp/index) system with an accelerating voltage of 50 $\mathrm{kV}$ and a current of $500 \mathrm{pA}$. The writing field was set to $600 \mu \mathrm{m} \times 600 \mu \mathrm{m}$ with 75000 dots, a beam diameter of $2 \mathrm{~nm}$ and an aperture size of $40 \mu \mathrm{m}$ to obtain a final resolution of $10 \mathrm{~nm}$. e-beam dose was varied by controlling the exposure time, ranging from $0.1 \mu \mathrm{s}$ to $2 \mu \mathrm{s}$ and no proximity effect correction was performed for the exposure. Resolution of $10 \mathrm{~nm}$ is referred to the minimum size of the radius achieved, that is due to a combined choice of exposure time, current and voltage. Once current and voltage are fixed, the longer the exposure time, the bigger the feature written on the sample will result. In principle, with low currents better resolution is achievable, but a balance should be found with the other parameters to focus correctly the E-beam machine (if the current is too low there is a high risk of mis-focusing the electron beam). Samples were developed in a solution of Methyl isobutyl ketone and isopropyl alcohol (MIBK:IPA 1:1) for 30 seconds and then immersed in DI-water for 30 seconds to stop the developing process. The samples were then blow-dried under a steady stream of $N_{2}$.

Reflectivity measurements We performed normal incidence reflectivity spectra by using NanoSpec 6100 Reflectometer (Nanometrics). Reflected light was collected with two different magnification objectives, 10x and 20x to ensure a two-step control of the consistency of the measurements. 
FDTD Simulations Simulations were carried out by using our in-house FDTD code NANOCPP. We investigated a geometry composed by a Silicon back reflector with a $350 \mathrm{~nm}$ layer of PMMA deposited on top. This last was patterned with an array of disordered holes, replicated directly from the design used as a mask for the nanofabrication. Simulations were implemented with different radius, filling fractions and e-beam doses to obtain reflectivity spectra consistent with the ones collected experimentally. In the simulations we used the dispersion curves of the materials from data available in the literature in the visible and near infrared spectral range (E.D. Palik, Handbook of optical constants of solids, Vol. 3, Academic press,1998).

Fitting of reflectivity spectra The normal reflectivity spectrum $R(\lambda)$ of a layer an interface Air/PMMA/Silicon was obtained from multilayer theory and read as follows:

$$
R(\lambda)=\frac{\left(1-n_{S i}\right)^{2} \cos ^{2} \frac{2 \pi d}{\lambda}+\left(\frac{n_{S i}}{n_{\mathrm{eff}}}-n_{\mathrm{eff}}\right)^{2} \sin ^{2} \frac{2 \pi d}{\lambda}}{\left(1+n_{S i}\right)^{2} \cos ^{2} \frac{2 \pi d}{\lambda}+\left(\frac{n_{S i}}{n_{\mathrm{eff}}}+n_{\mathrm{eff}}\right)^{2} \sin ^{2} \frac{2 \pi d}{\lambda}}
$$

with $n_{S i}(\lambda)$ the refractive index of Si and $d, n_{\mathrm{eff}}(\lambda)$ the thickness and refractive index of the PMMA layer, respectively. In our fitting procedure we employed the experimental available index of $\mathrm{Si}$ and $n_{\text {eff }}=\sqrt{1+\chi_{\text {eff }}}$ as a fitting parameter by using a two poles model with one resonance:

$$
\chi_{\mathrm{eff}}(\omega)=\frac{2 \rho_{0}}{3 \epsilon_{0} \hbar}\left|\boldsymbol{\mu}_{10}\right|^{2}\left(\frac{1}{\omega_{10}-\omega-i \gamma_{10}}+\frac{1}{\omega_{10}+\omega+i \gamma_{10}}\right)
$$

with parameters $\omega_{10}, \gamma_{10}$ and $a=\frac{2 \rho_{0}}{3 \epsilon_{0} \hbar}\left|\boldsymbol{\mu}_{10}\right|^{2}$ found by a swarm optimization routine. 
[1] Focusing in on applications. Nature Nanotechnology, 10:1 EP -, Jan 2015. Editorial.

[2] Robert W. Boyd. Nonlinear Optics, Third Edition. Academic Press, Inc., Orlando, FL, USA, 3rd edition, 2008.

[3] George Brewer. Electron-beam technology in microelectronic fabrication. Elsevier, 2012.

[4] Chuanlu Chen, Lin Zhou, Jianyu Yu, Yuxi Wang, Shuming Nie, Shining Zhu, and Jia Zhu. Dual functional asymmetric plasmonic structures for solar water purification and pollution detection. Nano Energy, 51:451-456, 2018.

[5] Tuck C Choy. Effective medium theory: principles and applications, volume 165. Oxford University Press, 2015.

[6] Zhaogang Dong, Jinfa Ho, Ye Feng Yu, Yuan Hsing Fu, Ramon Paniagua-Dominguez, Sihao Wang, Arseniy I Kuznetsov, and Joel KW Yang. Printing beyond srgb color gamut by mimicking silicon nanostructures in free-space. Nano letters, 17(12):7620-7628, 2017.

[7] Jason D Forster, Heeso Noh, Seng Fatt Liew, Vinodkumar Saranathan, Carl F Schreck, Lin Yang, Jin-Gyu Park, Richard O Prum, Simon GJ Mochrie, Corey S O'Hern, et al. Biomimetic isotropic nanostructures for structural coloration. Advanced Materials, 22(26-27):29392944, 2010.

[8] Henning Galinski, Gael Favraud, Hao Dong, Juan S Totero Gongora, Grégory Favaro, Max Döbeli, Ralph Spolenak, Andrea Fratalocchi, and Federico Capasso. Scalable, ultraresistant structural colors based on network metamaterials. Light: Science \& Applications, 6(5):e16233, 2017. 
[9] Juan S Totero Gongora, Andrey E Miroshnichenko, Yuri S Kivshar, and Andrea Fratalocchi. Anapole nanolasers for mode-locking and ultrafast pulse generation. Nature Communications, 8:15535, 2017.

[10] Wenchuang Hu, Koshala Sarveswaran, Marya Lieberman, and Gary H Bernstein. Sub-10 nm electron beam lithography using cold development of poly (methylmethacrylate). Journal of Vacuum Science \& Technology B: Microelectronics and Nanometer Structures Processing, Measurement, and Phenomena, 22(4):1711-1716, 2004.

[11] Mooseok Jang, Yu Horie, Atsushi Shibukawa, Joshua Brake, Yan Liu, Seyedeh Mahsa Kamali, Amir Arbabi, Haowen Ruan, Andrei Faraon, and Changhuei Yang. Wavefront shaping with disorder-engineered metasurfaces. Nature photonics, 12(2):84, 2018.

[12] Mikhail A Kats, Romain Blanchard, Patrice Genevet, and Federico Capasso. Nanometre optical coatings based on strong interference effects in highly absorbing media. Nature materials, 12(1):20, 2013.

[13] Mikhail A Kats and Federico Capasso. Ultra-thin optical interference coatings on rough and flexible substrates. Applied Physics Letters, 105(13):131108, 2014.

[14] Mohammadreza Khorasaninejad, Wei Ting Chen, Robert C Devlin, Jaewon Oh, Alexander Y Zhu, and Federico Capasso. Metalenses at visible wavelengths: Diffraction-limited focusing and subwavelength resolution imaging. Science, 352(6290):1190-1194, 2016. 
[15] Hyoki Kim, Jianping Ge, Junhoi Kim, Sung-eun Choi, Hosuk Lee, Howon Lee, Wook Park, Yadong Yin, and Sunghoon Kwon. Structural colour printing using a magnetically tunable and lithographically fixable photonic crystal. Nature Photonics, 3(9):534-540, 2009.

[16] Yuri Kivshar. All-dielectric meta-optics and non-linear nanophotonics. National Science Review, 5(2):144-158, 2018.

[17] Anders Kristensen, Joel KW Yang, Sergey I Bozhevolnyi, Stephan Link, Peter Nordlander, Naomi J Halas, and N Asger Mortensen. Plasmonic colour generation. Nature Reviews Materials, 2:16088, 2016.

[18] Karthik Kumar, Huigao Duan, Ravi S Hegde, Samuel CW Koh, Jennifer N Wei, and Joel KW Yang. Printing colour at the optical diffraction limit. Nature nanotechnology, 7(9):557-561, 2012.

[19] Lianlin Li, Tie Jun Cui, Wei Ji, Shuo Liu, Jun Ding, Xiang Wan, Yun Bo Li, Menghua Jiang, Cheng-Wei Qiu, and Shuang Zhang. Electromagnetic reprogrammable coding-metasurface holograms. Nature communications, 8(1):197, 2017.

[20] Peining Li, Irene Dolado, Francisco Javier Alfaro-Mozaz, Fèlix Casanova, Luis E Hueso, Song Liu, James H Edgar, Alexey Y Nikitin, Saül Vélez, and Rainer Hillenbrand. Infrared hyperbolic metasurface based on nanostructured van der waals materials. Science, 359(6378):892-896, 2018.

[21] Dianmin Lin, Pengyu Fan, Erez Hasman, and Mark L Brongersma. Dielectric gradient metasurface optical elements. Science, 345(6194):298-302, 2014. 
[22] Changxu Liu, Andrea Di Falco, D Molinari, Yasser Khan, Boon S Ooi, Thomas F Krauss, and Andrea Fratalocchi. Enhanced energy storage in chaotic optical resonators. Nature Photonics, 7(6):473-478, 2013.

[23] Sofia Magkiriadou, Jin-Gyu Park, Young-Seok Kim, and Vinothan N. Manoharan. Absence of red structural color in photonic glasses, bird feathers, and certain beetles. Phys. Rev. E, 90:062302, Dec 2014.

[24] Sergii Morozov, Michele Gaio, Stefan A Maier, and Riccardo Sapienza. Metal-dielectric parabolic antenna for directing single photons. Nano letters, 18(5):3060-3065, 2018.

[25] Yusuke Nagasaki, Masafumi Suzuki, and Junichi Takahara. All-dielectric dual-color pixel with subwavelength resolution. Nano letters, 17(12):7500-7506, 2017.

[26] Xingjie Ni, Naresh K Emani, Alexander V Kildishev, Alexandra Boltasseva, and Vladimir M Shalaev. Broadband light bending with plasmonic nanoantennas. Science, 335(6067):427427, 2012.

[27] Andrew R Parker and Helen E Townley. Biomimetics of photonic nanostructures. Nature nanotechnology, 2(6):347-353, 2007.

[28] Fei Qin, Lu Ding, Lei Zhang, Francesco Monticone, Chan Choy Chum, Jie Deng, Shengtao Mei, Ying Li, Jinghua Teng, Minghui Hong, et al. Hybrid bilayer plasmonic metasurface efficiently manipulates visible light. Science advances, 2(1):e1501168, 2016.

[29] Racheli Ron, Emir Haleva, and Adi Salomon. Nanoporous metallic networks: Fabrication, optical properties, and applications. Advanced Materials, page 1706755, 2018. 
[30] Christian Schlickriede, Naomi Waterman, Bernhard Reineke, Philip Georgi, Guixin Li, Shuang Zhang, and Thomas Zentgraf. Imaging through nonlinear metalens using second harmonic generation. Advanced Materials, 30(8):1703843, 2018.

[31] Amr M Shaltout, Jongbum Kim, Alexandra Boltasseva, Vladimir M Shalaev, and Alexander V Kildishev. Ultrathin and multicolour optical cavities with embedded metasurfaces. Nature communications, 9(1):2673, 2018.

[32] Yichen Shen, Veronika Rinnerbauer, Imbert Wang, Veronika Stelmakh, John D Joannopoulos, and Marin Soljacic. Structural colors from fano resonances. ACS Photonics, 2(1):27-32, 2015.

[33] Shawn J Tan, Lei Zhang, Di Zhu, Xiao Ming Goh, Ying Min Wang, Karthik Kumar, ChengWei Qiu, and Joel KW Yang. Plasmonic color palettes for photorealistic printing with aluminum nanostructures. Nano letters, 14(7):4023-4029, 2014.

[34] Jérémie Teyssier, Suzanne V Saenko, Dirk Van Der Marel, and Michel C Milinkovitch. Photonic crystals cause active colour change in chameleons. Nature communications, 6:6368, 2015.

[35] Andreas Tittl, Aleksandrs Leitis, Mingkai Liu, Filiz Yesilkoy, Duk-Yong Choi, Dragomir N Neshev, Yuri S Kivshar, and Hatice Altug. Imaging-based molecular barcoding with pixelated dielectric metasurfaces. Science, 360(6393):1105-1109, 2018. 
[36] Xiaofei Zang, Fengliang Dong, Fuyong Yue, Chunmei Zhang, Lihua Xu, Zhiwei Song, Ming Chen, Pai-Yen Chen, Gerald S Buller, Yiming Zhu, et al. Polarization encoded color image embedded in a dielectric metasurface. Advanced Materials, 30(21):1707499, 2018.

[37] Xiaolong Zhu, Christoph Vannahme, Emil Højlund-Nielsen, N Asger Mortensen, and Anders Kristensen. Plasmonic colour laser printing. Nature nanotechnology, 11(4):325-329, 2016.

[38] Xiaolong Zhu, Wei Yan, Uriel Levy, N Asger Mortensen, and Anders Kristensen. Resonant laser printing of structural colors on high-index dielectric metasurfaces. Science Advances, 3(5):e1602487, 2017. 

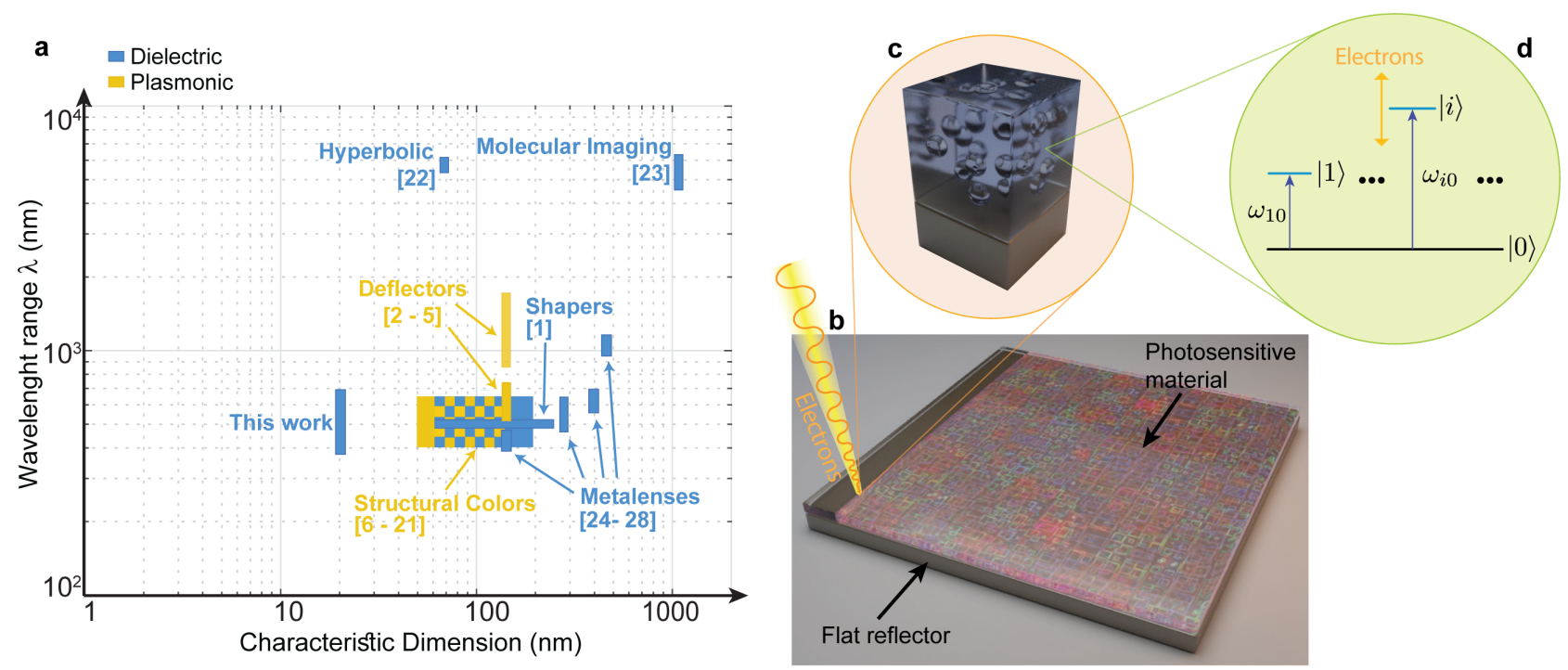

Figure 1: Broadband light control with free-electron metasurfaces: the idea. (a) State of the art in metasurface fabrication. Comparison between current resolution and working wavelength ranges achieved with dielectric structures, plasmonic structures and in this work. (b-d) Fabrication steps with (b) free-electrons irradiation, (c) material removal, and (d) shifts of the electronic energy transitions in the remaining irradiated volume, imprinting a desired material refractive index $n$ and extinction coefficient $k$. 
a

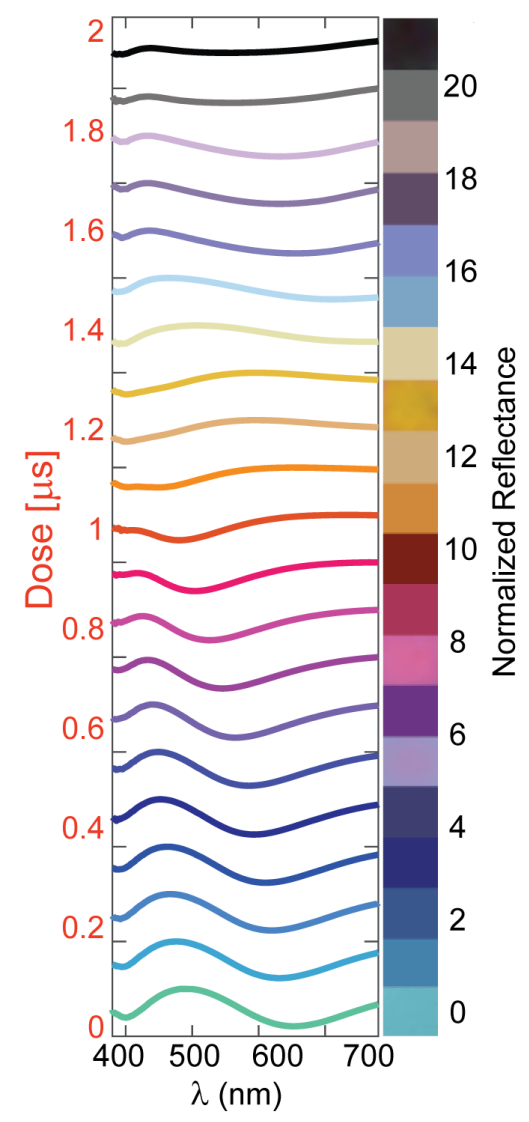

b

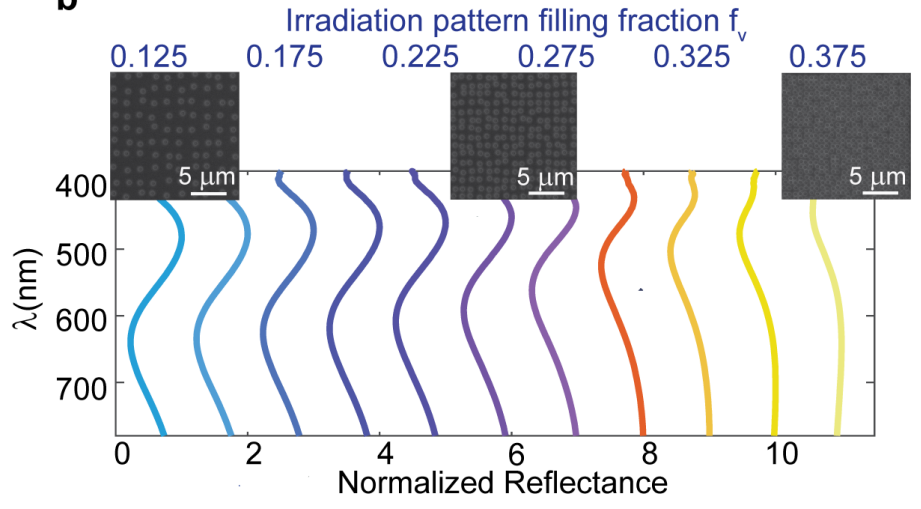

C

Normalized Reflectance
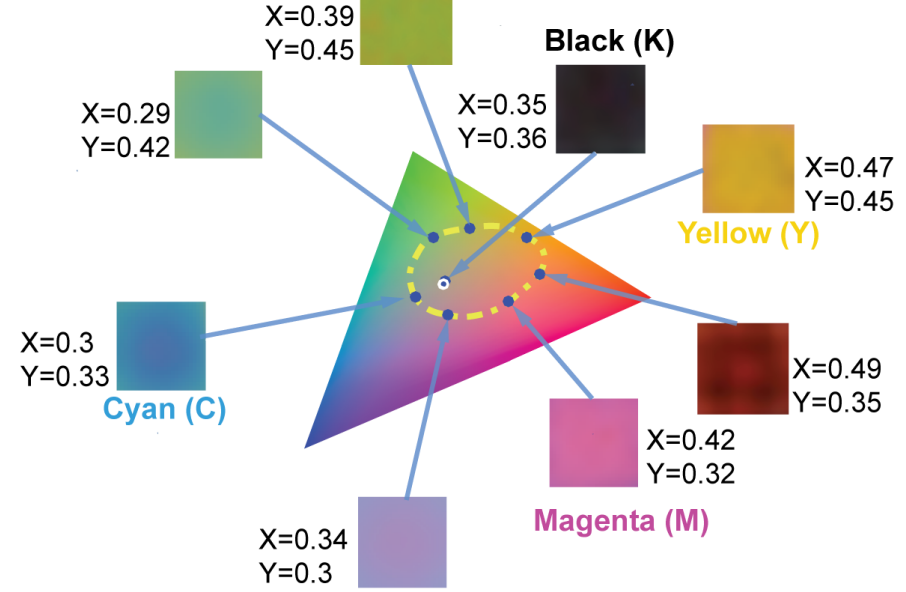

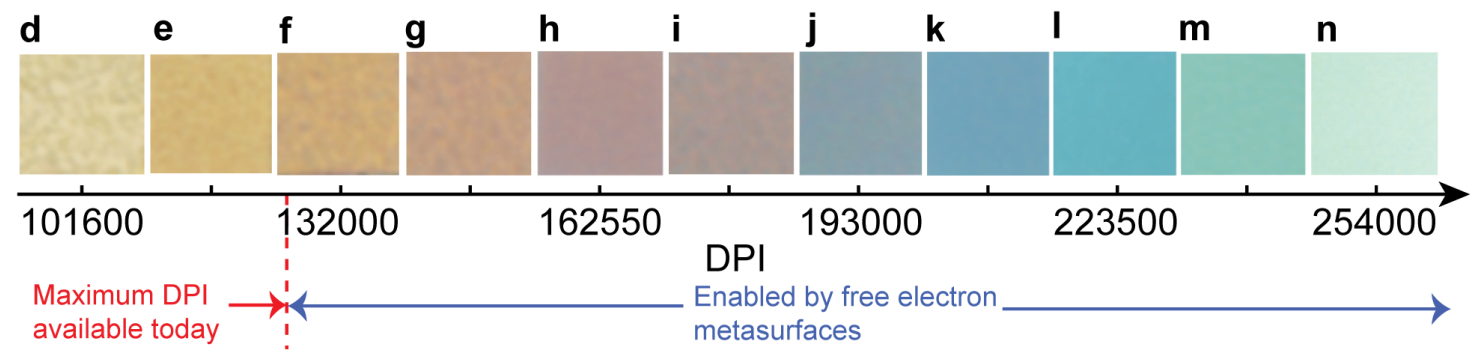

Figure 2: Structural colors characterization. (a), (b) Experimental reflectivity spectra for increasing free-electrons exposure time and different radiation patterns. The colors of the reflectivity spectra show the visual appearance of the metasurface. (c) Resulting chromaticity diagram, with insets showing images of experimental samples and the corresponding coordinates in the XYZ system. (d-n) Colors printed on $20 \mu \mathrm{m}$ by $20 \mu \mathrm{m}$ squares at increasing resolution. 

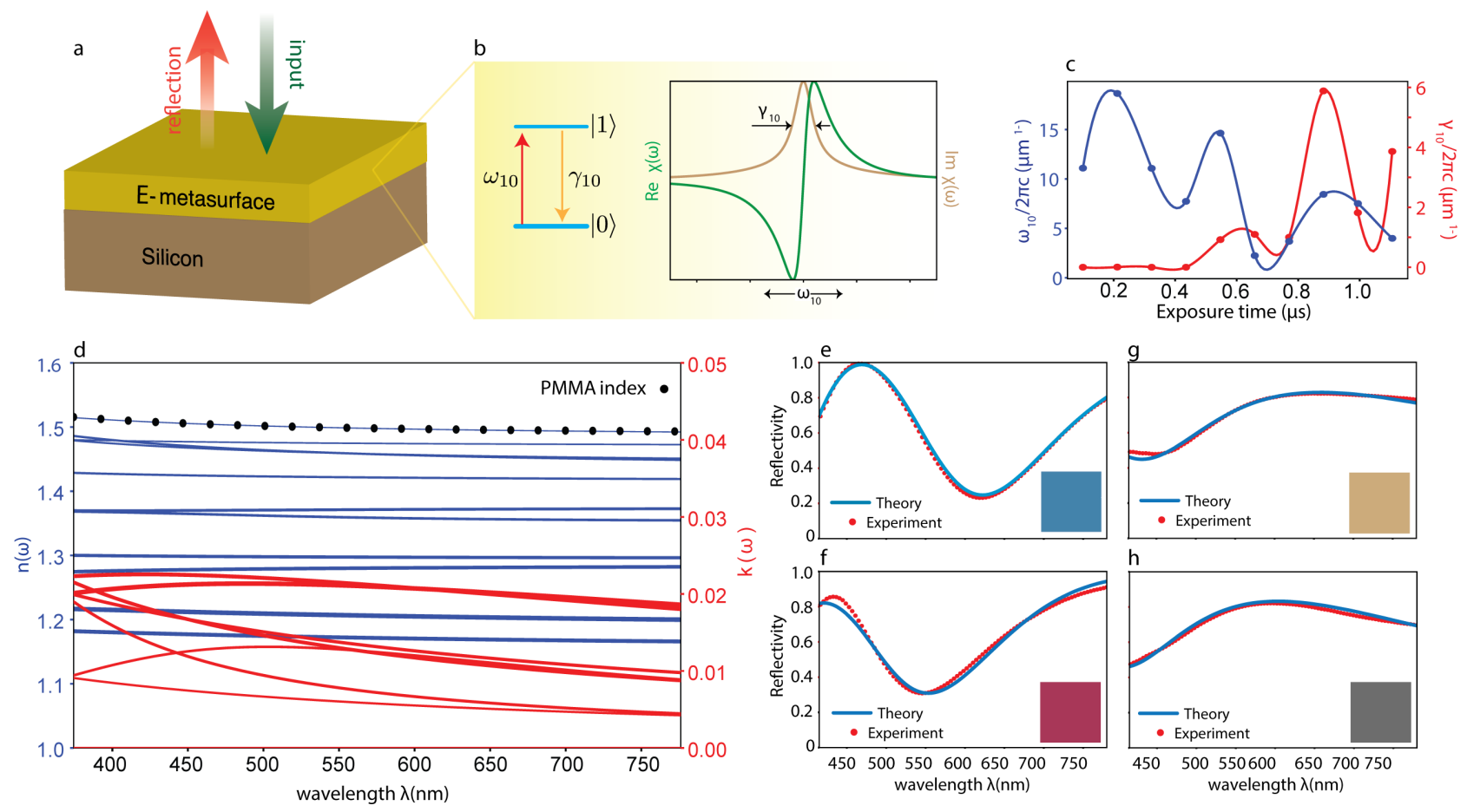

Figure 3: Theoretical analysis. (a) Sketch of the analyzed system composed by a Si substrate with on top a PMMA layer characterized by a single electronic transition with tunable resonant frequency $\omega_{10}$ and damping $\gamma_{10}$. The latter are evaluated from experimental reflectivity spectra at normal incidence. (c) Plot of $\omega_{10}$ and $\gamma_{10}$ versus e-beam dose and (d) resulting dispersion curve of refractive index $n(\omega)$ and extinction coefficient $k(\omega)$. The increased exposure time is represented with an increasing thickness of the lines representing the index $n$ and extinction $k$. (ef) Comparison between experimental spectra (red markers) and theoretical predictions (blue line) from Eq. 2, insets of the corresponding colors are presented for each experimental spectrum. 


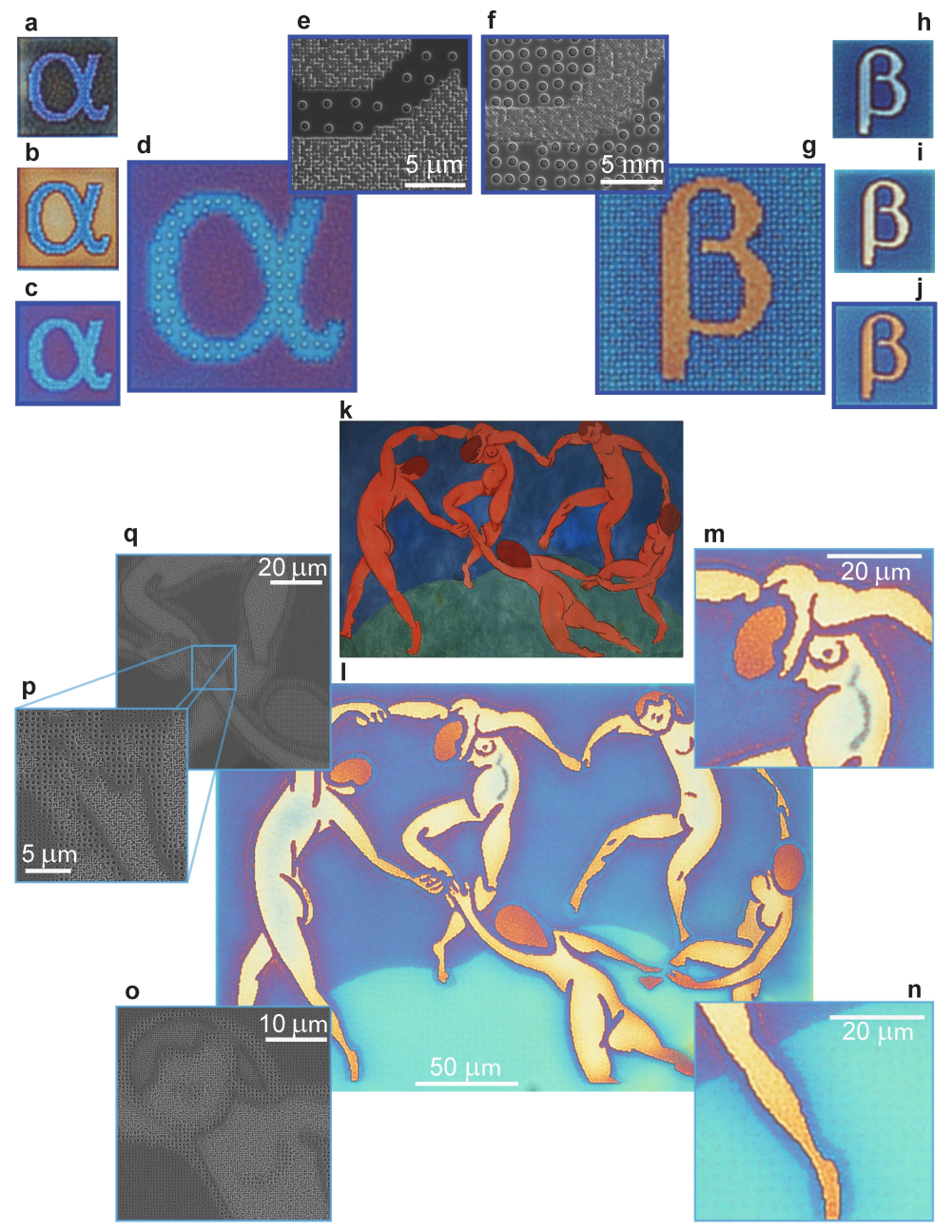

Figure 4: Nanoprinting applications. Top view images of the letters ad) $\alpha$ and fj) $\beta$ with different colors and backgrounds obtained with a filling fraction $f_{v}=0.075$ (letter) and $f_{v}=0.250$ (background) in (ad), and $f_{v}=0.3$ (letter) and $f_{v}=0.175$ (background) in (gj). Different colors are obtained at different exposure times: a,g) at $t=2 \mu \mathrm{s}, \mathrm{b}, \mathrm{h})$ at $t=1 \mu \mathrm{s}$, and c,d,i,j) at $t=0.2 \mu s$. e,f) SEM details of panels (d) and (g), respectively. k) Original painting "La Danse" by Matisse (Henri Matisse, La Dance, 1910. Copyright Succession H. Matisse/2019, ProLitteris, Zurich). lq) Reproduction of the painting "La Danse" by Matisse with an average resolution of 100000 DPI and a maximum resolution of 119250 DPI. m,n) Optical microscope magnification of the fabricated sample with 40x objective. oq) SEM images of the sample. 